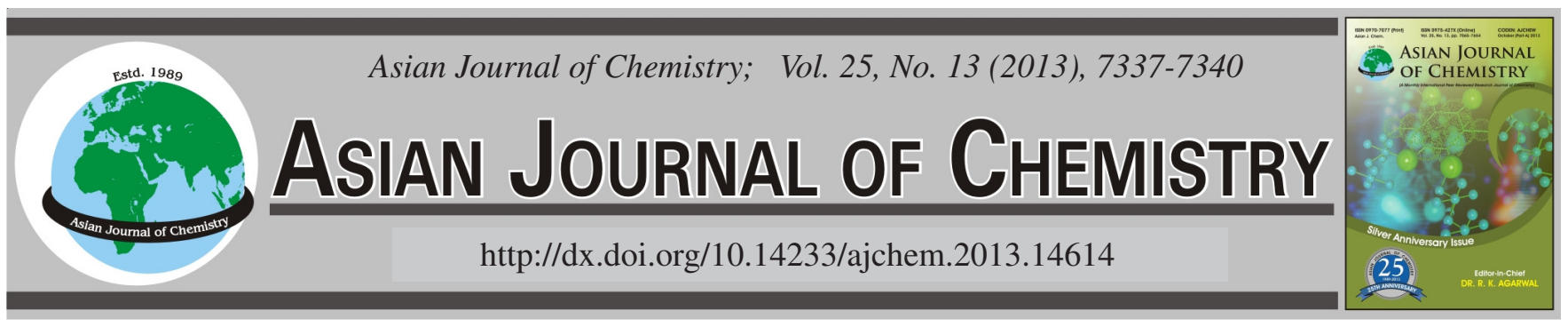

\title{
Determination of Degree of Crystallinity of Pantoprazole Sodium Sesquihydrate by Thermogravimetric Analysis
}

\author{
M. Arthanareeswari ${ }^{*}$, A. Ravikiran, P. Kamaraj and Ch. Praveen
}

Department of Chemistry, SRM University, SRM Nagar, Kattankulathur-603 203, India

*Corresponding author: Fax: +91 44 277453903; Tel: +91 44 27455855; E-mail: arthanareeswari@gmail.com

\begin{abstract}
Determination of degree of crystallinity by the available general comparative analytical techniques require reference materials of single phase or mixture of known amount of phases to compare with. Current study explores thermogravimetric analysis (TGA) as a tool to determine the absolute degree of crystallinity of a class of Active Pharmaceutical Ingredients, and this method uses no reference material for comparison. Pantoprazole sodium sesquihydrate, an API has been used to demonstrate the application. Hi-Res dynamic TGA experimentation is used to accurately determine the weight loss due to bound water and the same is confirmed by VT-PXRD experiments. The degree of crystallinity is calculated using the amount of bound water. The result $(84.7 \%)$ was found to be comparable with the value $(82.4 \%)$, calculated from PXRD profiles.
\end{abstract}

Key Words: Degree of crystallinity, Hi-Res-dynamic TGA, Pantoprazole sodium sesquihydrate.

ᄂ - - - - - - - - - - - - - - - - - - - - - - - - - - - -

\section{INTRODUCTION}

The common crystalline forms available for any active pharmaceutical ingredient (API) are polymorphs or solvates. Polymorphs have same chemical entity but differ in internal crystal structure and therefore, posses different physico-chemical properties ${ }^{1-5}$. Solvates, also called as pseudo polymorphs, are crystalline adducts containing solvent molecules within the crystal structure, in either stoichiometric or non-stoichiometric proportions, giving rise to unique differences in physical and pharmaceutical properties. If the incorporated solvent is water, a solvate is termed as hydrate ${ }^{6}$. It has been estimated that approximately one-third of the pharmaceutically active substances are capable of forming crystalline hydrates ${ }^{7}$. The water because of its small size can easily fill structural voids and because of its multidirectional bonding capability, is also ideal for linking of drug molecules into stable crystal structures $^{8}$. Crystallization plays a critical role in controlling the crystalline form and the distribution in size and shape of the drug $^{9-11}$. A crystalline phase is created as a consequence of molecular aggregation processes in solution that lead to the formation of nuclei, which achieve a certain size during the nucleation phase to enable growth into macroscopic crystals to take place during the growth phase ${ }^{6}$.

A perfectly ordered crystal lattice with every molecule in its expected lattice position is an ideal that is ever achieved. The other extreme is the amorphous state, in which a solid contains the maximum possible density of imperfections (defects of various dimensionalities), such that all long-range order is lost while only the short-range order, imposed by its nearest molecular neighbors, remains. Real crystals lie somewhere between these two extremes. A crystal's position on a scale bounded by these two extremes is termed its crystallinity ${ }^{12}$. In reality, a powder probably contains particles with different degrees of crystallinity, just as it may contain particles with different sizes and shapes. The lower the crystallinity of a particle, the greater its enthalpy and entropy. The increase in enthalpy is never totally compensated by the increase in entropy. However, the Gibbs free energy, which reflects the balance between them, actually increases. Hence, the lower the crystallinity of a particle and consequently the greater it's amorphous character, the greater it's apparent intrinsic solubility, dissolution rate and reactivity, but the lower its stability. Polymorphism and its variation in the degree of crystallinity in a pharmaceutical substance may exhibit physicochemical differences that impacts therapeutic, manufacturing, commercial and legal levels. Because of the great relevance of these properties, crystallinity is an important property and requires measurement by a suitable method ${ }^{12}$. Various methods are available for the measurement of crystallinity like X-ray diffraction, calorimetry, density measurements, infrared spectroscopy, Raman spectroscopy, solid-state nuclear magnetic resonance, etc. ${ }^{13}$. 
In present study, the weight loss due to bound water (bound to crystal lattice) and surface water of pantoprazole sodium sesquihydrate API have been determined with the help of Hi-Res TGA; the same has been confirmed by variable temperature PXRD and water by Karl Fisher method ${ }^{15}$. The amount of weight loss due to bound water can be attributed to crystalline portion of the solid; degree of crystallinity is calculated against theoretical weight loss value for $100 \%$ crystalline pantoprazole sodium sesquihydrate, which is $6.24 \%$ (w/w), calculated from the molecular formula. Amorphous and crystalline fractions were also estimated from the PXRD profile $^{16}$ and the results are compared.

\section{EXPERIMENTAL}

Pantoprazole sodium sesquihydrate (CAS Registry No: 138786-67-1, molecular weight 432.36) is an antiulcerative. Pantoprazole sodium sesquihydrate is a sodium salt of 5(difluoro-methoxy)-2-[[(3,4-dimethoxy-2-pyridinyl)methyl]sulfinyl]-1H-benzimidazole. Chemical structure of pantoprazole sodium sesquihydrate is represented in Fig. 1.<smiles></smiles>

Fig. 1. Chemical structure of pantoprazole sodium sesquihydrate

Reagents like sodium tartrate dihydrate (water standard for volumetric K.F titration, Merck KGaA, Damstadt, Germany), Pyridine-free Karl-Fisher single solution (Merck Specialties Private Limited, Worli, Mumbai, India) and Chromatographic grade Methanol (Merck Specialties Private Limited, Mumbai, India) are used for the determination of water content by K.F autotitator.

Karl-Fisher titration: The analysis was performed using KF Autotitator (841 Titrando, Metrohm, Switzerland). The determination of total moisture by Karl Fisher titration is a calculation based on the concentration of Iodine in the KF titrating reagent (i.e., titer) and the amount of Karl-Fisher reagent consumed in the titration. The end-point of the titration is determined by the dead-stop end-point method. About $40 \mathrm{~mL}$ of fresh methanol is taken into dry titration vessel. $100.2 \mathrm{mg}$ of the pantoprazole sodium sesquihydrate API is accurately weighed and transferred into well conditioned methanol in the solution in the vessel titrated with the Karl-Fisher reagent and the water content was calculated from the end point obtained. Karl-Fisher factor used for the calculations is determined by using sodium tartrate dihydrate.

Powder X-ray diffraction (PXRD): Powder X-ray diffractometer used is of $\theta-\theta$ geometry with Lynx Eye detector. The instrument make and model is Bruker AXS and D8 ADVANCE, respectively. Peak position accuracy was checked using NIST Standard Reference material 1976a (Corundum). The X-ray tube is operated at a voltage of $40 \mathrm{kV}$ and current of $40 \mathrm{~mA}$. Each diffraction profile is collected in continuous mode and in the scan range of $3-45^{\circ} 2 \theta$ with a step size of $0.01^{\circ} 2 \theta$ and with a time per step of 0.4 s. PMMA (poly- methyl methacrylate) sample holder $(\varnothing 25 \mathrm{~mm})$ is used for the specimen preparation. Top loading method is followed. Data acquisition and analysis were performed on BRUKER's XRD Commander and Diffrac plus EVA softwares, respectively.

Variable temperature PXRD (VT-PXRD): Hot Humidity Chamber (\# MRI-7009000), HOT HUMIDITY controller (\# ANS-SYCOSHOT) has been used only to heat the sample. The diffraction profiles were collected in situ for every $10^{\circ} \mathrm{C}$ interval from $30-120^{\circ} \mathrm{C}$. The temperature program is given in such a way that at each temperature point the specimen is equilibrated for about $5 \mathrm{~min}$, before starting the PXRD scan. The PXRD profiles are collected in the range 4.6-40 $2 \theta$ with a step size and time per step of $0.02^{\circ} 2 \theta$ and $0.16 \mathrm{~s}$, respectively, the approximate run time of each scan is $5 \mathrm{~min}$. Stainless steel sample holder of hot stage accessory is used for the specimen preparation. Top loading method is followed.

Thermogravimetric analysis: The experiments were performed on TGA Q5000 of TA Instruments (Lukens Drive, Delaware, USA), in Hi-Res (High Resolution) mode with dynamic test procedure under the nitrogen gas purge at a flow of $40 \mathrm{~mL} / \mathrm{min}$ for balance and $60 \mathrm{~mL} / \mathrm{min}$ for sample. Instrument was calibrated for temperature with Nickel (supplied by the TA instruments) and TGA balance was calibrated with certified weights (Denver Instrument-calibration weights, Denver, Colorado). The experimental conditions for dynamic test procedure, in which the samples were analyzed on the Aluminum pan, with a heating rate of $30^{\circ} \mathrm{C} / \mathrm{min}$ up to $125^{\circ} \mathrm{C}$ and resolution number of 4 with sensitivity value of 1 . Data acquisition and analysis were performed using Universal Analysis 2000 software (TA Instruments).

\section{RESULTS AND DISCUSSION}

Analysis by Karl Fisher autotitration and powder X-ray diffraction (PXRD): Water content determined by Karl-fisher autotitator found to be $6.65 \%$ (w/w). PXRD profile of the initial material of pantoprazole sodium sesquihydrate (Fig. 2a) has been used to calculate approximate value of degree of crystallinity ${ }^{16}$. An important note is that the values obtained by this method are relative but not absolute.

$$
\text { Degree of crystallinity }=\frac{100 \times A}{(A+B-C)}
$$

$A=$ Total area of the peaks arising from diffraction from the crystalline fraction of the sample, as represented in Fig. 2b, the observed value for the sample is $276.2 \mathrm{Cps} \mathrm{X} \operatorname{deg}, \mathrm{B}=$ total area below area A, as represented in Fig. 2c. $\mathrm{C}=$ background area (due to air scattering, fluorescence, equipment, etc.), as represented in Fig. 2 d.

Denominator $(\mathrm{A}+\mathrm{B}-\mathrm{C})$ indicates the total area contributed from both crystalline and amorphous portions, the value is obtained from the integration of total area under the diffraction profile after removing the background area $(\mathrm{C})$. The denominator value was found to be $335.3 \mathrm{Cps} \mathrm{X}$ deg.

Substituting the values of $\mathrm{A}$ and $\mathrm{A}+\mathrm{B}-\mathrm{C}$ in to the above equation, the value of degree of crystallinity by PXRD was found to be $82.4 \%$.

Analysis by VT-PXRD: Overlaid diffraction pattern of PXRD profiles collected at every $10^{\circ} \mathrm{C}$ interval from $30-120^{\circ} \mathrm{C}$ is shown in Fig. 3, indicating the diffraction pattern is remained 

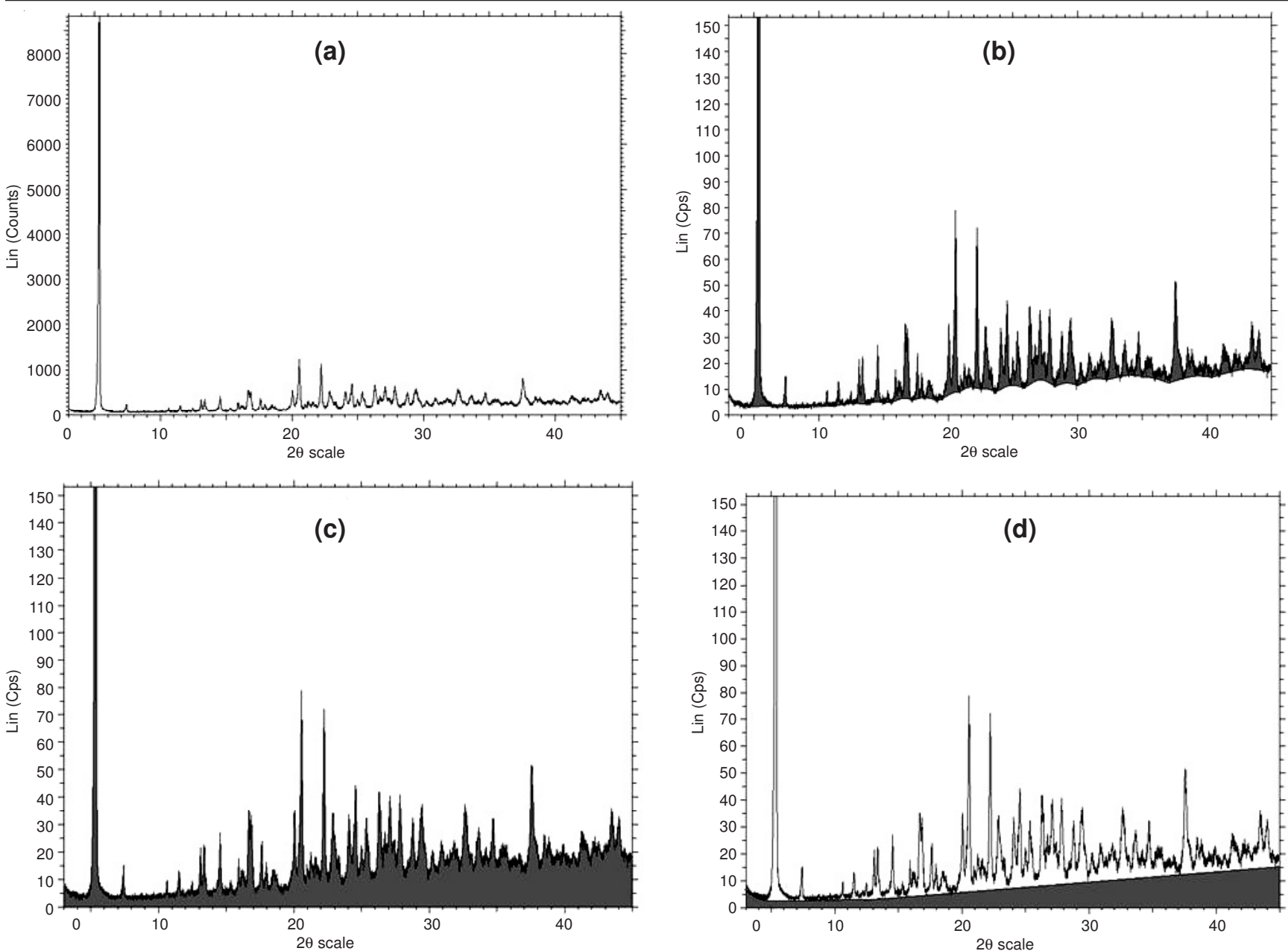

Fig. 2. (a) Powder diffraction pattern of pantoprazole sodium sesquihydrate. (b) Darken area of powder diffraction pattern of pantoprazole sodium sesquihydrate is pictorial representation of area "A". (c) Darken area of powder diffraction pattern of pantoprazole sodium sesquihydrate is pictorial representation of area "B". (d) Darken area of powder diffraction pattern of pantoprazole sodium sesquihydrate is pictorial representation of Area "C"

unchanged till the temperature about $100^{\circ} \mathrm{C}$, at $c a .110^{\circ} \mathrm{C}$ the pattern show a change in the intensity of the peaks, finally at $120^{\circ} \mathrm{C}$ the pattern completely changed to broad amorphous hallow. The study indicates the thermal events that occurred in that temperature range $\left(100-120^{\circ} \mathrm{C}\right)$ resulted in collapse of crystal lattice, due to bound water loss.

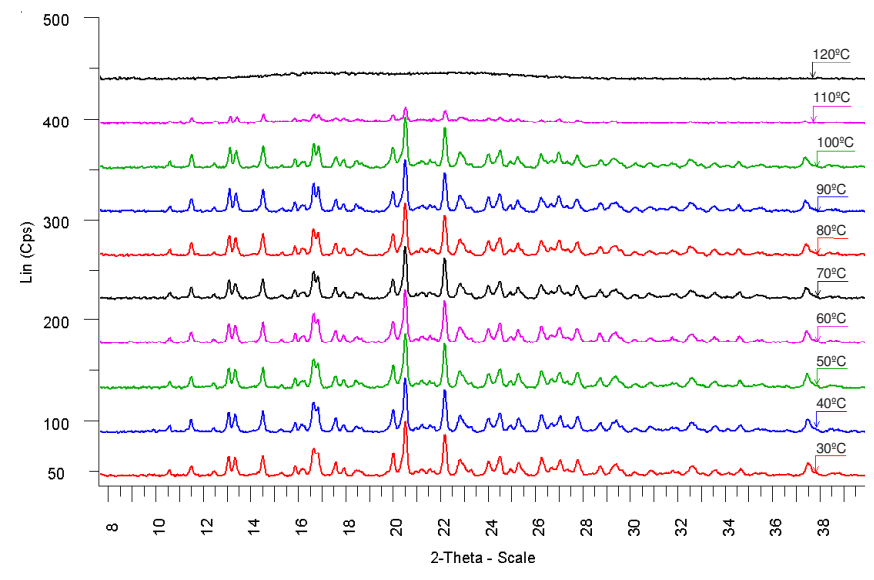

Fig. 3. Overlaid diffraction pattern of PXRD profiles collected at every 10 ${ }^{\circ} \mathrm{C}$ interval from $30-120{ }^{\circ} \mathrm{C}$
Analysis by thermogravimetric analyzer: Typical TGA thermogram of the sample along with its 1st derivative curve is shown in the Fig. 4. Total weight loss integrated from 29.6$108.13{ }^{\circ} \mathrm{C}$ is $6.534 \%(\mathrm{w} / \mathrm{w})$, which is comparable with the water content determined by K.F $(6.65 \%)$. 1st derivative curve (short dashed line) of TGA thermogram shows that there is a sharp inflection onset at $97.22^{\circ} \mathrm{C}$ and endset at $c a .108 .13{ }^{\circ} \mathrm{C}$. In the similar temperature range VT-PXRD study indicated an abrupt change in the diffraction profile. The integrated weight loss during this inflation is found to be $5.288 \%$; this weight loss corresponds to the water present in the crystal lattice, i.e., bound water weight loss.

Calculations: Molecular weight of pantoprazole sodium sesquihydrate is 432.36 , where $1.5 \mathrm{~mol}$ water contributes to $6.245 \%(\mathrm{w} / \mathrm{w})$. In another words, if the given sample is a perfectly ordered crystal lattice with every molecule of drug along with water molecules (in 1:1.5 ratio) present in its expected lattice position, the total weight of bound water should be $c a .6 .245 \%$. The observed bound water weight loss obtained from Hi. Res TGA experiments is ca. $5.288 \%$. Therefore,

The degree of crystallinity of the given powder is

$$
=\frac{(5.288 \times 100)}{6.245}=84.7 \%
$$


TABLE-1

DEGREE OF CRYSTALLINITY CALCULATED FROM HI. RES. TGA EXPERIMENTS AND PXRD

\begin{tabular}{|c|c|c|c|c|c|}
\hline $\begin{array}{l}\text { S. } \\
\text { No. }\end{array}$ & Compound name & $\begin{array}{l}\text { Theoretical \% (wt/wt) } \\
\text { of water calculated } \\
\text { from molecular formula } \\
\text { (A) }\end{array}$ & $\begin{array}{l}\text { Wt \% loss due to bound } \\
\text { water detrmined from Hi. } \\
\text { Res. TGA experiment } \\
\text { (B) }\end{array}$ & $\begin{array}{l}\text { Degree of crystallinity } \\
\text { calculated from Hi. } \\
\text { Res. TGA experiments } \\
\text { (BX100/A) }\end{array}$ & $\begin{array}{l}\text { Degree of } \\
\text { crystallinity } \\
\text { calculated from } \\
\text { PXRD profile }\end{array}$ \\
\hline 1 & $\begin{array}{l}\text { Pantoprazole sodium sesquihydrate } \\
\qquad \mathrm{C}_{16} \mathrm{H}_{14} \mathrm{~F}_{2} \mathrm{~N}_{3} \mathrm{NaO}_{4} \mathrm{~S}: 1.5 \mathrm{H}_{2} \mathrm{O}\end{array}$ & 6.245 & 5.288 & 84.7 & 82.4 \\
\hline
\end{tabular}

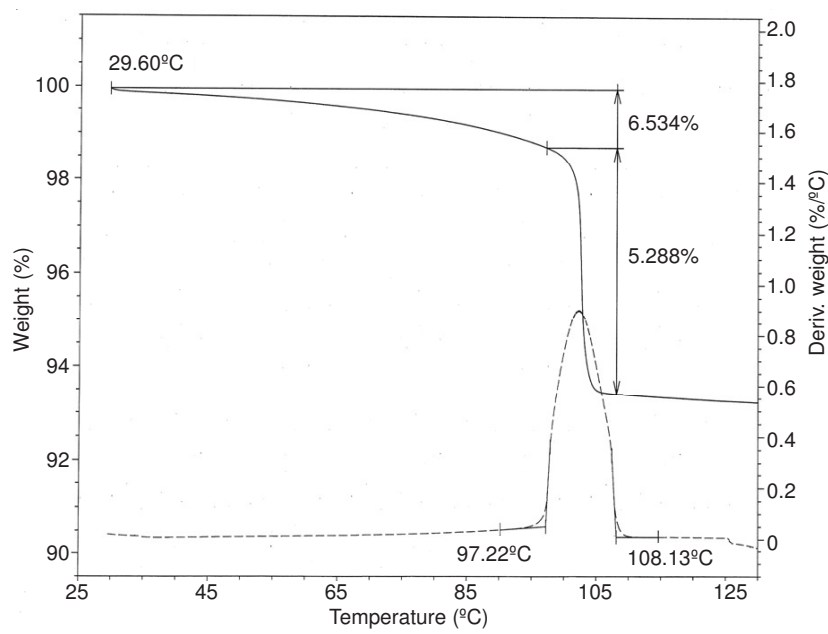

Fig. 4. TGA thermogram of pantoprazole sodium sesquihydrate API

The results are summarized in Table- 1 .

\section{Conclusion}

The current study shows light on the applicability of thermogravimetric analysis as a tool for the determination of absolute value of degree of crystallinity, which is an important property of pharmaceutical solids in the drug product performance point of view. The basic idea of the study is that the total weight loss obtained in a thermogravimetric analysis is a combination of bound and unbound water/solvent, the amount of bound water is a measure of crystalline hydrate portion. The approach would help in understanding the crystallinity of solids of stoichiometric hydrates. The value of degree of crystallinity obtained from TGA found to be comparable with the approximate value calculated from powder X-ray diffraction profile. The absolute value of degree of crystallinity obtained by this TGA experiment can be used as a reference value for other comparative quantitative phase analysis by different techniques like powder X-ray diffraction, spectral methods, thermal methods, etc.

\section{REFERENCES}

1. M. Kuhnert-Brandstatter, Thermomicroscopy in the Analysis of Pharmaceuticals, Pergamon, Oxford (1971).

2. L. Borka and J.K. Haleblian, Acta Pharm. Jugosl., 40, 71 (1990).

3. H.G. Brittain, Polymorphism in Pharmaceutical Solids, Marcel Dekker Inc. New York (1999).

4. L. Borka, Pharm. Acta. Helv., 66, 16 (1991).

5. D. Giron, Thermochim. Acta, 248, 1 (1995).

6. S.R. Vippaguntaa and H.G. Brittain, Adv. Drug Delivery Rev., 48, 3 (2001).

7. H.P. Stahl, In ed.: D.D. Braimar, The Problems of Drug Interactions with Excipients, Towards Better Safety of Drugs and Pharmaceutical Products, Elsevier North-Holland Bio-medical Press, pp. 265-280 (1980).

8. S.R. Byrn, R.R. Pfeiffer and J.G. Stowell, Solid State Chemistry of Drugs, SSCI, West Lafayette, IN (1999).

9. D.J.W. Grant, In ed.: H.G. Brittain, Theory and Origin of Polymorphism, Polymorphism in Pharmaceutical Solids, Marcel Dekker, New York, Vol. 95, pp. 1-33 (1999).

10. N. Rodriguez-Hornedo and D. Murphy, J. Pharm. Sci., 88, 651 (1999).

11. J.W. Mullin, Crystallization, Butterworth Heinemann, Oxford (1993).

12. General Ch. 696. Crystallinity Determination by Solution Calorimetry, The United States Pharmacopeial Convention, USP34-NF29, p. 270 (2011).

13. N. Chieng, T. Rades and J. Aaltonen, J. Pharm. Biomed. Anal., 55, 618 (2011).

14. A.F. Barnes, M.J. Hardy and T.J. Lever, J. Therm. Anal., 40, 499 (1993).

15. General Ch. 921, Water Determination, The United States Pharmacopeial Convention, USP34-NF29, p. 407 (2011).

16. General Ch. 941, Characterization of Crystalline and Partially Crystalline Solids by X-Ray Powder Diffraction (XRPD), The United States Pharmacopeial Convention, USP34-NF29, p. 410 (2011). 